\title{
Design highlights and status of the LHAASO project
}

\author{
Huihai $\mathrm{He}^{\mathbf{1}}$ for the LHAASO collaboration \\ Institute of High Energy Physics, Chinese Academy of Sciences \\ Beijing 100049, China \\ E-mail: hhheihep.ac.cn
}

The Large High Altitude Air Shower Observatory (LHAASO) plans to build a hybrid extensive air shower (EAS) array with an area of $1 \mathrm{~km}^{2}$ at an altitude of $4410 \mathrm{~m}$ a.s.l. in Sichuan province, China, aiming for very high energy gamma ray astronomy and cosmic ray physics around the spectrum knees. With a sensitivity of $1 \%$ Crab unit, the LHAASO will survey the entire northern sky for gamma ray sources with full duty cycle. The spectra of all sources in its field of view will be measured simultaneously over a wide energy range from $300 \mathrm{GeV}$ to $1 \mathrm{PeV}$. This measurement will offer a great opportunity for identifying cosmic ray origins among the sources. The LHAASO is also equipped with Cherenkov/fluorescence telescopes and in-filled burst detector array, so it will serve as an effective detector for energy spectral measurement of different mass groups of cosmic rays.

In this contribution, the design highlights and status of the LHAASO project are presented.

The 34th International Cosmic Ray Conference

30 July- 6 August, 2015

The Hague, The Netherlands

${ }^{1}$ Speaker 


\section{Introduction}

The Large High Altitude Air Shower Observatory (LHAASO) project [1] plans to build a hybrid extensive air shower (EAS) array with an area of $1 \mathrm{~km}^{2}$ at an altitude of 4,410 m a.s.l. in Sichuan province, China, aiming for very high energy gamma ray astronomy and study of cosmic rays with energies in $10^{13}-10^{18} \mathrm{eV}$, where the cosmic ray spectrum knees around $10^{15.5} \mathrm{eV}$ and $10^{17.5} \mathrm{eV}$ are located. With a sensitivity of $1 \%$ Crab unit, the LHAASO will survey the northern hemisphere for gamma ray sources with full duty cycle. The spectra of all sources in its field of view will be measured simultaneously over a wide energy range from $100 \mathrm{GeV}$ to $1 \mathrm{PeV}$. This measurement will offer a great opportunity for identifying cosmic ray origins among the sources. The LHAASO is also equipped with Cherenkov/fluorescence telescopes and an in-filled shower core detector array, so it will serve as an effective detector for energy spectral measurement of different mass groups of cosmic rays.

In this contribution, the design highlights and status of the LHAASO project are presented.

\section{The LHAASO detector arrays}

The LHAASO consists of an extensive air shower array covering an area of $1 \mathrm{~km}^{2}(\mathrm{KM} 2 \mathrm{~A})$, 75,000 $\mathrm{m}^{2}$ water Cherenkov detector array (WCDA), an in-filled shower core detector array (SCDA) and 12 wide-field air Cherenkov/fluorescence telescopes (WFCTA), as shown in Fig.1. An integral sensitivity of $1 \% \mathrm{Crab}$ unit can be reached at $3 \mathrm{TeV}$ and $50 \mathrm{TeV}$. Its differential sensitivity to gamma ray sources is also shown in Fig.1.
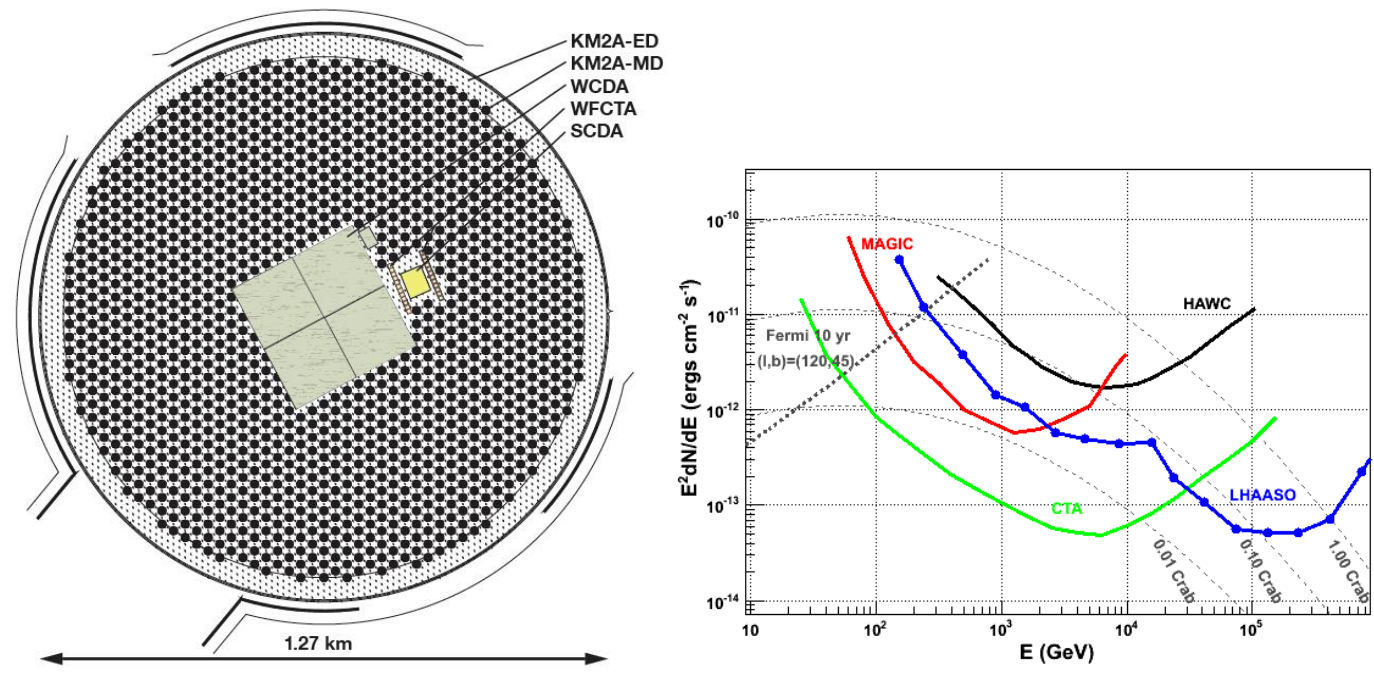

Fig.1 LHAASO schematic (left) and differential sensitivity (right) to gamma ray sources compared to other experiments/projects [2-6]. The Crab SED used here is got by fitting measurements of Fermi, ARGO-YBJ, Tibet AS $\gamma$, MAGIC, VERITAS, HESS, HEGRA and Whipple:

$$
3.18 \times 10^{-11} \times(\mathrm{E} / \mathrm{TeV})^{-(2.536+0.210 \times \log 10(\mathrm{E} / \mathrm{TeV}))} \times(\mathrm{E} / \mathrm{TeV})^{2} \times 1.6\left(\mathrm{erg} \mathrm{cm}^{-2} \mathrm{~s}^{-1}\right)
$$

\subsection{KM2A}

An exposure of approximately $2000 \mathrm{~km}^{2} \mathrm{hour} / \mathrm{year}$ is required to observe in one year more than 10 gamma rays with energies above $100 \mathrm{TeV}$ from a source with a flux of $1 \%$ Crab unit. Furthermore, with an expected number of gamma rays as low as the order of 10, a background- 
free observation to these rays is mandatory. Cosmic nuclei dominate the background with a flux of 3 to 4 orders of magnitude higher than that of gamma rays, so a detector working in this energy region should reach a background rejection power of $10^{4}$ or even higher while its acceptance to gamma rays should be high enough (e.g. >90\%) after background rejection. An imaging air Cherenkov telescope array, even with 100 telescopes to cover an area of $10 \mathrm{~km}^{2}$, can only reach a sensitivity of about $10 \%$ Crab unit at $100 \mathrm{TeV}$ because of its low duty cycle (10\%-15\%) and background rejection power (the order of $10^{2}$ ). In LHAASO, KM2A has a full duty cycle (about 2000 hours of exposure to Crab Nebula per year) and covers an area of $1 \mathrm{~km}^{2}$ with 5195 scintillation detectors (EDs, $1 \mathrm{~m}^{2}$ each) in a triangular grid with a spacing of $15 \mathrm{~m}$. The EDs provide the main useful information on the injected primary cosmic rays or gamma rays, such as energy and direction, while shower muons are detected by 1171 muon detectors (MDs, $36 \mathrm{~m}^{2}$ each) in a triangular grid with a spacing of $30 \mathrm{~m}$. Considering that gamma showers are muonpoor, measuring the numbers of electromagnetic components and muons in an EAS can highly discriminate original gamma rays from the cosmic nuclei. With the large-area MD array $(>40,000$ $\mathrm{m}^{2}$ in total area) in LHAASO, cosmic nuclei background will be rejected to a level of $10^{-4}$ at 50 $\mathrm{TeV}$ and even $10^{-5}$ at higher energies [7].

\subsubsection{KM2A-ED}

An ED (Fig.2) consists of 4 plastic scintillation tiles $(100 \mathrm{~cm} \times 25 \mathrm{~cm} \times 2.5 \mathrm{~cm}$ each) covered by $5 \mathrm{~mm}$-thick lead plates to absorb low energy charged particles in showers and convert shower gammas into electron-positron pairs. Sixteen WLS fibers ( $2.7 \mathrm{~m}$-long and $1.5 \mathrm{~mm}$ in diameter) are embeded in 32 grooves (each $1.8 \mathrm{~mm}$ in depth and $1.6 \mathrm{~mm}$ in width) of each tile to collect scintillation lights generated by charged particles interjecting in it and guide the scintillation lights to a 1.5-inch photomultiplier tube (PMT). Each PMT is supplied by a high-voltage module with a temperature coefficient of $<0.01 \% /{ }^{\circ} \mathrm{C}$, thus the high voltage of each PMT can be adjusted independently.

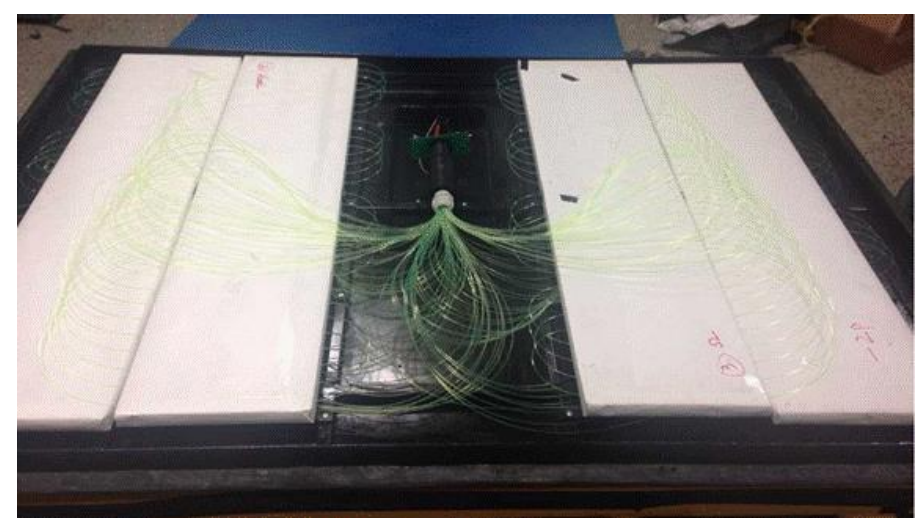

Fig.2 An ED prototype.

The detection efficiency of a ED to MIPs is required to be $>95 \%$, with a time resolution of better than $2 \mathrm{~ns}$ and a signal charge resolution of $<25 \%$ (defined as the sigma/mean of the signal charge distribution). The dynamic range for each ED is designed to be 1-10,000 particles, with the upper limit corresponding to the large number of shower secondaries near the shower core of the highest energy (up to $100 \mathrm{PeV}$ ) hadrons to be detected. The signal attenuation due to aging is required to be less than $20 \%$ in 10 years. 


\subsubsection{KM2A-MD}

To achieve the main physical purposes, area of a LHAASO MD unit is optimized to $36 \mathrm{~m}^{2}$ by balancing the total area and spacing of MDs in simulation [7]. To measure accurately the number of muons detected, an MD should have sufficiently good detection efficiency ( $>95 \%)$ and signal charge resolution ( $<25 \%$, defined as the sigma/mean of the signal charge distribution) of single muons. The purity of the number of muons detected in hadronic showers should be $>95 \%$. With a total area of $>40,000 \mathrm{~m}^{2}$, the total background muon rate of LHAASO MDs could reach as high as $10^{7} \mathrm{~Hz}$, which corresponds to 1 muon in 100ns. These background muons can be effectively rejected if a coincidence time window of $\pm 30 \mathrm{~ns}$ around a shower front is used in the data analysis [7]. Thus, a time resolution of $<10 \mathrm{~ns}$ is fairly good, while MDs do not participate in the triggering and reconstruction of shower direction. The lower limit of MD's dynamic range is decided by single muon signals, while the upper limit corresponds to the large number of muons near the shower core of the highest energy (up to $100 \mathrm{PeV}$ ) hadrons to be detected. Finally, the long-term stability is also very important since LHAASO will operate for more than 10 years, in which the signal attenuation should be less than $20 \%$.

The design with water Cherenkov detector underneath dirt is chosen for LHAASO MDs (Fig.3). A water bag with a diameter of $6.8 \mathrm{~m}$ is used to enclose pure water. An 8-inch photomultiplier tube (PMT) sits at top center of the water bag and looks downwards through a highly transparent window into the water. The water bag is contained in a cylindrical concrete tank with an inner diameter of $6.8 \mathrm{~m}$. The thickness of the overburden dirt and the water depth are tuned by simulation to be $2.5 \mathrm{~m}$ and $1.2 \mathrm{~m}$ respectively. The liner reflectivity is required to be higher than $95 \%$, and the water absorption length should be longer than $50 \mathrm{~m}$.

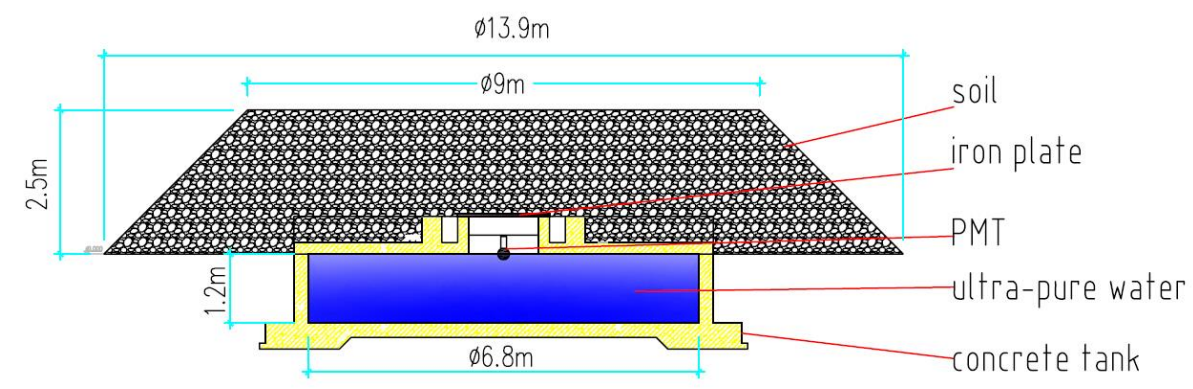

Fig.3 Schematic of LHAASO muon detector.

The liner consists of separate laminates by proper processing method, and the laminates comprise four layers of co-extruded materials. The inner layer is made of Tyvek 1082D (DuPont) which is an opaque material with excellent strength, good flexibility, and high diffuse reflectivity for near-UV lights. Tyvek is non-woven material made of high-density polyethylene, which can minimize the possibility of chemicals leaching into the water volume. The middle two layers are made of LDPE film with good strength and outstanding chemical resistance. PP film is chosen as the outer layer because of its hard-wearing.

The PMT window is made of EVA plastic which is formed according to shape of PMT photocathode. The EVA plastic has a relatively high UV transmittance and can be easily molded. Furthermore, Silicon oil is added between PMT and the window for optical coupling. 


\subsection{WCDA}

The WCDA focuses on surveying the northern sky for steady and transient sources from 100 $\mathrm{GeV}$ to $20 \mathrm{TeV}$, with a very high background rejection power and a good angular resolution. The WCDA consists of 3 water ponds with a total area of $75,000 \mathrm{~m}^{2}$, and the effective water depth is $4.4 \mathrm{~m}$ (Fig.4). Each water pond is divided into hundreds of cells with size $5 \mathrm{~m} \times 5 \mathrm{~m}$, partitioned by black plastic curtains to prevent penetration of the lights yielded in neighboring cells. An 8-9 inch PMT sits at the bottom center of each cell, looking upward to collect Cherenkov lights generated by shower secondary particles in water.

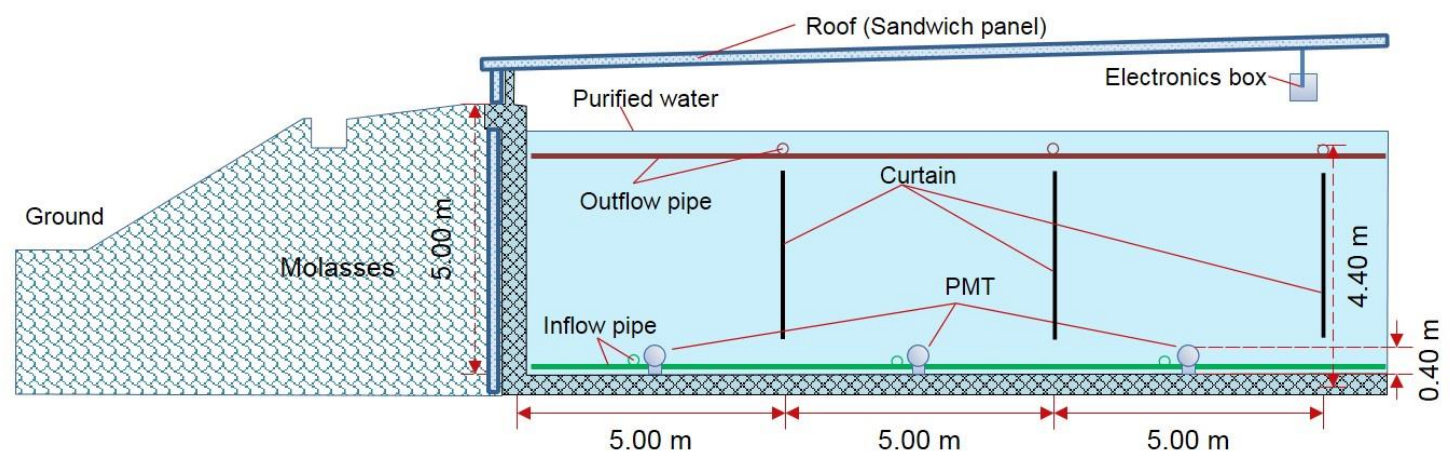

Fig.4 WCDA schematic.

The construction of the pond is explained as follows. The bank and the floor of the water pond is made up of reinforced concrete or common concrete, with plastic liner paved in the inner side for water-proofing. The roof of the pond is structured with insulation sandwich steel plates, supported by pillars and steel frames.

Water transparency and statbility is critical for the experiment. In the very beginning, the pond is filled with water drilled from a well or river, after purified by a industrial standard purification system. As a part of the water circulation system, two layers of pipe webs is set up in the top and bottom of the pond, to fill and drill the pond water all the time during the whole operation period of the experiment, and a purification system, made up of 3 levels of fine filters ( $5 \mu \mathrm{m}, 1 \mu \mathrm{m}$, and $0.22 \mu \mathrm{m}$ ), and two levels of UV radiation lights (254 nm and $185 \sim \mathrm{nm}$ ), works in the middle of the flow to remove or destruct particles, microbes and organics. With these measures, the absorption length of water at blue wavelength can maintain in a level of greater than $15 \mathrm{~m}$, meeting the requirement of the experiment.

\subsection{WFCTA}

The WFCTA is composed of 12 wide-field-of-view Cherenkov/fluorescence telescopes. Each telescope (see Fig.5 for a prototype telescope) consists of a housing and mechanical system spherical light collector of about $4.7 \mathrm{~m}^{2}$ and focal plane camera of $32 \times 32$ pixels made of a PMT array. Each pixel has a FOV of $\sim 0.5^{\circ}$ and is read out by FEE and digitization using a FADC at 50 $\mathrm{MHz}$ on the back board of the camera. The camera is calibrated by a UV LED light source mounted at the center of the mirror during operation. The slow-control and monitoring system are completely remotely accessible. The whole telescope is installed in a shipping box whose doors are remotely opened and closed. The whole telescope can be manually elevated up-and-down. The entrance is sealed by using glass window which is cleaned every month before operation. 
With 12 telescopes, a field-of-view of $180^{\circ}$ in azimuth and $25^{\circ}$ in elevation can be achieved as show in Fig.5.
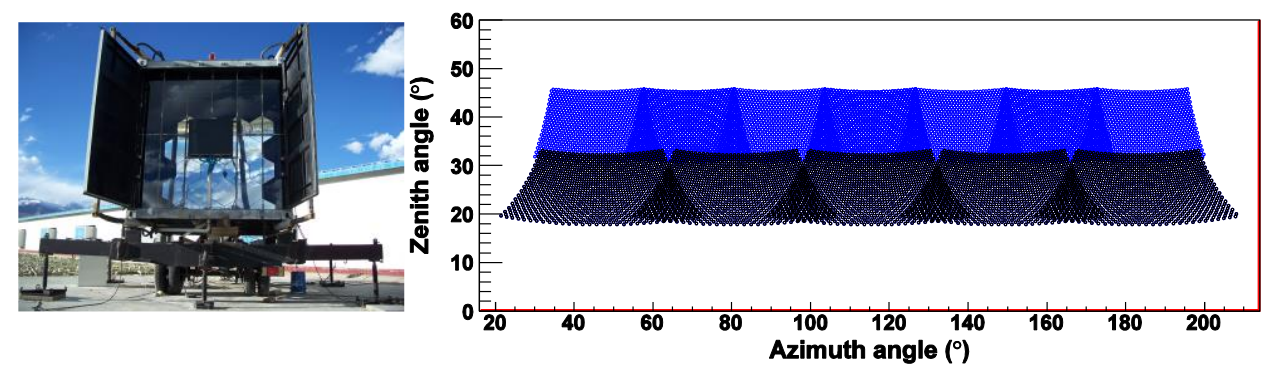

Fig.5 A prototype telescope of WFCTA (left) and the field-of-view of 12 telescopes (right).

\subsection{Extension to UHE for second knee}

To extend the spectrum to higher energies and make a connection with experiments, such as TA and Auger, the wide FOV telescopes will be re-arranged to measure shower fluorescence light and monitoring the space above the ground array from a distance of 4 or $5 \mathrm{~km}$. The detector configuration is shown in Fig.6, in which the main detector array is composed of 12 telescopes covering elevations from $3^{\circ}$ to $59^{\circ}$ and 2 telescopes (the existing prototypes), covering elevations from $10^{\circ}$ to $24^{\circ}$, observe showers from perpendicular directions. Showers above $100 \mathrm{PeV}$ will be detected stereoscopically to maintain a high resolution of Xmax. Muon content and Xmax are used for composition measurement around the second knee of the spectrum.

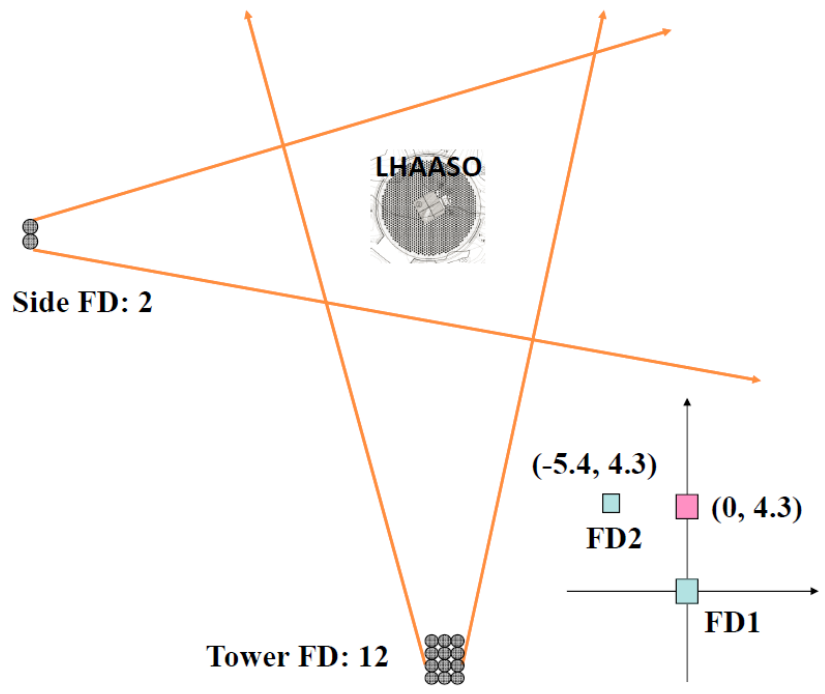

Fig.6 Re-configuration of WFCTA for hybrid measurement of showers with fluorescence lights and muon contents of showers.

\section{LHAASO Electronics and Data Acquisition System}

All signals of the LHAASO detectors come from PMTs. The signal arriving time and charge are the major measures. The former represents the arriving time of shower secondary particles and is mainly used for primary direction reconstruction, thus it is crucial for astronomy-oriented detectors of LHAASO, namely KM2A and WCDA. For these detectors an overall time resolution of $2 \mathrm{~ns}$ is required, thus a resolution of $0.5-1 \mathrm{~ns}$ is required for the corresponding time-to-digital converters (TDCs). KM2A and WCDA electronics use FPGA-based TDCs to assign to each 
detector hit a time stamp. Since the LHAASO detectors are distributed in an area as large as 1 $\mathrm{km} 2$ and signal digitization is done by the front-end electronics, synchronization over all the TDC clocks is the key of the TDC system, for which the White Rabbit (WR) protocol developed by CERN is adopted. The accuracy of the baseline design of WR is $<1 \mathrm{~ns}$, while LHAASO needs a better one (0.5ns for KM2A and 0.2ns for WCDA). Collaborating with the CERN WR group, a group from Qinghua University of China is working on upgrading the WR baseline design to meet the requirement of LHAASO [8-9]. The clock synchronization system of the LHAASO also promises hybrid measurement of showers by detector arrays triggered independently.

For the LHAASO detectors, signal charge is a measure of the number of shower particles. It is required by both astronomy and CR oriented detectors of the LHAASO, mainly for shower energy determination. The LHAASO electronics integrate the signals in different time windows for different detectors. Each LHAASO detector array covers a large energy range and the dynamic range of detector unit usually reaches more than 3 orders of magnitude. Special design of PMT HV divider is developed to enlarge the PMT dynamic range, and furthermore, dynode readout is implemented to supplement the dynamic range of anode readout. This makes the electronics much simpler and easier to reach the required dynamic range. Considering the high electronics density and high altitude environment, ASIC for LHAASO PMT charge readout is under development to reduce the electronics heat dissipation and cost. For the LHAASO muon detectors and the WFCTA (working in fluorescence mode), the signal time duration is about hundreds of ns or even wider. FADC-based waveform digitization will be implemented for charge measurement.

All of the LHAASO signals will be digitized at the front-end. Digitized instead of analog signals will be transferred through TCP/IP to the processing center where soft-triggering and event building will be done via a farm of thousands of CPU cores. Raw data will be immediately transferred to the IHEP computer center for further analysis.

\section{LHAASO prototype arrays}

Basing on the design of the detectors, electronics, WR-based clock distribution and DAQ, aprototype arrays about $1 \%$ of LHAASO have been constructed at Yangbajing Cosmic Ray Observatory, which include a KM2A prototype with 42 EDs and 2 MDs (Fig.7-left), a WCDA prototype with $3 \times 3$ units (Fig.7-right), 2 WFCTA prototype telescopes (Fig.5). All the prorotype arrays have been in operation for more than 2 years and the performances fully meet the design requirements, see [10-15] for details.
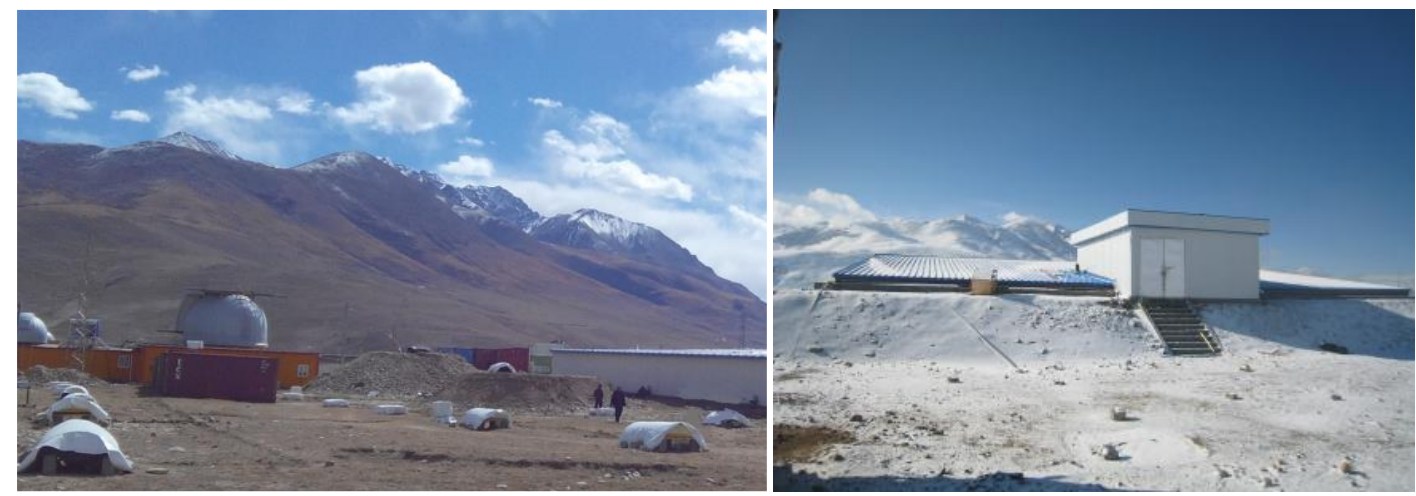

Fig.7 Prototype arrays of KM2A (left) and WCDA (right).

\section{Conclusion}


The LHAASO is designed to fulfill the physical goals in gamma ray astronomy and cosmic ray physics. Prototype detector arrays of about 1\% LHAASO are designed and built at the Yangbajing Cosmic Ray Observatory and used to coincidentally measure cosmic rays with the ARGO-YBJ experiment. The LHAASO field preparation will start in 2015 and detector construction is expected to start in 2016 and finish in 2020. According to the construction schedule, 1/4 of LHAASO will be put into operation and produce physical data in 2018.

\section{Acknowledgement}

This work is supported in China by NSFC (NO.11205165, NO. 11375210, No.11375224, No.11405181, No.11475190), the Chinese Academy of Sciences, Institute of High Energy Physics, the Key Laboratory of Particle Astrophysics, CAS and in Italy by the Istituto Nazionale di Fisica Nucleare (INFN).

\section{References}

[1] Z. Cao., LHAASO Coll., Frascati Physics Series, Vol. 58(2014)

[2] http://www.slac.stanford.edu/exp/glast/groups/canda/lat_Performance.htm

[3] J. Aleksic,et al., arxiv:1409.5594v3 (2015), accepted for publication in Astroparticle Physics , in press

[4] https://portal.cta-observatory.org/Pages/CTA-Performance.aspx

[5] K. Bernlöhr, et al., Astroparticle Physics 43 (2013) 171-188

[6] A.U. Abeysekara, et al., Astroparticle Physics 50-52 (2013) 26 - 32

[7] S. Cui et al., LHAASO Coll., Astroparticle Physics, 54 (2014) 86-92

[8] Q. Du, G. Gong, W. Pan et al., Nuclear Instruments and Methods in Physics Research, A732 (2013) $488-492$

[9] H. Li, G. Gong, W. Pan et al., IEEE TRANSACTIONS ON NUCLEAR SCIENCE, VOL. 62, NO. 3, JUNE 2015

[10] J. Liu et al., LHAASO Coll., Chinese Physics C, Vol. 38, No. 2 (2014) 026001

[11] J. Zhao et al., LHAASO Coll., Chinese Physics C, Vol. 38, No. 3 (2014) 036002

[12] X. Zuo et al., LHAASO Coll., Nuclear Instruments and Methods in Physics Research, A789 (2015) $143-149$

[13] B. Gao et al., LHAASO Coll., Chinese Physics C, Vol.38, No.2 (2014)

[14] H. Li et al., LHAASO Coll., Chinese Physics C,Vol.38, No.1 (2014)

[15] B. Bartoli et al., Chinese Physics C, Vol. 38, 045001 (2014) 